\title{
From evolving artificial gene regulatory networks to evolving spiking neural networks for pattern recognition
}

\author{
Ahmed Abdelmotaleb ${ }^{1,2^{*}}$, Maria Schilstra ${ }^{1}$, Neil Davey ${ }^{1}$, Volker Steuber ${ }^{1}$, Borys Wróbel ${ }^{2,3}$ \\ From Twenty Second Annual Computational Neuroscience Meeting: CNS*2013 \\ Paris, France. 13-18 July 2013
}

The GReaNs platform (which stands for Gene Regulatory evolutionary artificial Networks) is an artificial life software platform that has been previously shown to allow the evolution of networks for signal processing and animat control [1]. The structure of the resulting network in this model is encoded in a linear genome, without imposing any restrictions on the size of the genome or the size of the network. We extended this model to encode spiking neural networks [4]. In previous work using GReaNs [1], each node in the artificial network has been considered to be an analog of a biological transcriptional unit. However, the nodes can also be seen as artificial neurons. Our extension has introduced to GReaNs two models of spiking neurons (LIF: leaky integrate and fire neurons with a fixed threshold [2] and AdEx: adaptive-exponential integrate and fire [3]). Here we use GReaNs for the evolution of spiking networks for pattern recognition. The genetic algorithm used in GReaNS is based on evolving the number of the neurons and the weights of the connections, but the synaptic delays are constant. We evolved a network that generates spikes at a maximum firing rate when the network is presented with one spike from five input neurons in a specific order, and that stays silent when the order is reversed. We also used three inputs, each with one spike, requiring the network to fire at a maximum rate for a specific temporal order of spikes and to remain silent for all the other permutations. In further work, we plan to investigate the generalization abilities of the evolved networks and the robustness of their behaviour to noise. GReaNs can export a PyNN script, which allows the simulation of an evolved network on different backends, including neuromorphic real-time hardware for real time simulation.

\section{Author details}

'Biocomputation Research Group, University of Hertfordshire, Hatfield, Herts AL10 9AB, UK. 'Evolutionary Systems Laboratory, Adam Mickiewicz University, Poznan, 61-712, Poland. '3ystems Modeling Laboratory, IOPAN, Sopot, 81712 , Poland.

Published: 8 July 2013

\section{References}

1. Wróbel B, Joachimczak M: Using GReaNs (Genetic Regulatory evolving artificial Networks) for 3-dimensional Asymmetrical Pattern Formation and Morphogenesis. Proceedings of DevLeaNN: Workshop on Development and Learning in Artificial Neural Networks 2011, 26-28.

2. Dayan P, Abbott LF: Theoretical neuroscience. Boston MIT Press; 2001

3. Gerstner W, Brette R: Adaptive exponential integrate-and-fire model as an effective description of neuronal activity. J Neurophysiol 2005, 94:3637-3642.

4. Wróbel B, Abdelmotaleb A, Joachimczak M: Evolving spiking neural networks in the GReaNs (Gene Regulatory Evolving Artificial Networks) platform. EvoNet2012: Evolving Networks, from Systems/Synthetic Biology to Computational Neuroscience Workshop at Artificial Life XIII 2012, 19-22.

5. Davison AP, Brüderle D, Eppler JM, Kremkow J, Muller E, Pecevski DA, Perrinet $L$ and Yger P: PyNN: a common interface for neuronal network simulators. Front Neuroinform 2009, 2-11.

doi:10.1186/1471-2202-14-S1-P423

Cite this article as: Abdelmotaleb et al:: From evolving artificial gene regulatory networks to evolving spiking neural networks for pattern recognition. BMC Neuroscience 2013 14(Suppl 1):P423.

\footnotetext{
* Correspondence: ahmed@evosys.org

'Biocomputation Research Group, University of Hertfordshire, Hatfield, Herts AL10 9AB, UK

Full list of author information is available at the end of the article
} 